\title{
Impact of radial extracorporeal shock wave therapy in post-laminectomy epidural fibrosis in a rat model
}

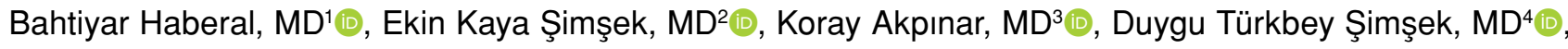 \\ Fikret Şahintürk, MD ${ }^{5}$ \\ 1Department of Orthopaedics and Traumatology, Başkent University Faculty of Medicine, Ankara, Turkey \\ ${ }^{2}$ Department of Orthopaedic and Traumatology, Beypazarı State Hospital, Ankara, Turkey \\ ${ }^{3}$ Department of Orthopaedics and Traumatology, Başkent University Faculty of Medicine, Ankara, Turkey \\ ${ }^{4}$ Department of Pathology, Başkent University Faculty of Medicine, Ankara, Turkey \\ ${ }^{5}$ Department of Neurosurgery, Başkent University Faculty of Medicine, Ankara, Turkey
}

Laminectomy is a widely used surgical method in the treatment of various spinal disorders, particularly lumbar spinal stenosis. Laminectomy membrane is formed in the area of bone defect due to bleeding in the deep layer of the paravertebral muscles after laminectomy. ${ }^{[1,2]}$ Epidural fibrosis develops as a result of the exaggerated healing tissue in this area, causing adhesions in the dura mater and nerve roots, and is one of the most important causes of failed back surgery syndrome caused by recurrent pain after surgery. ${ }^{[3,4]}$ Revision surgeries performed due to recurrent pain after surgery may cause complications such as epidural bleeding, nerve root damage and dural lacerations, and worsen the clinical outcome. Therefore, the number of studies investigating

Received: July 07, 2020

Accepted: October 02, 2020

Published online: January 06, 2021

Correspondence: Bahtiyar Haberal, MD. Başkent Üniversitesi Tıp Fakültesi, Ortopedi ve Travmatoloji Anabilim Dalı, 06490 Bahçelievler, Ankara, Türkiye.

E-mail: bahtiyarhaberal@hotmail.com

\section{Doi: $10.5606 /$ ehc. 2021.77870}

Citation: Haberal B, Kaya Simşek E, Akpınar K, Türkbey Simşek D, Sahintürk F. Impact of radial extracorporeal shock wave therapy in post-laminectomy epidural fibrosis in a rat model. Jt Dis Relat Surg 2021;32(1):162-169.

(02021 All right reserved by the Turkish Joint Diseases Foundation

This is an open access article under the terms of the Creative Commons Attribution-NonCommercial License, which permits use, distribution and reproduction in any medium, provided the original work is properly cited and is not used for commercial purposes (http://creativecommons.org/licenses/by-nc/4.0/).

\section{ABSTRACT}

Objectives: This study aims to investigate the effect of radial extracorporeal shock wave therapy (rESWT) treatment in the prevention of epidural fibrosis after laminectomy in rats.

Materials and methods: Eighteen 16-month-old male Sprague-Dawley rats weighing $300 \mathrm{~g}$ were used in this experimental study between November 2019 and February 2020. The rats were randomly divided into two groups as the control group (L3-L4 total laminectomy without any treatment) and the study group (L3-L4 total laminectomy plus rESWT). The rats were sacrificed at the postoperative sixth week and the lumbar spine was excised en bloc, fixed, and decalcified. Sections were stained with hematoxylin-eosin to evaluate epidural fibrosis, acute inflammation, chronic inflammation, and vascular proliferation.

Results: The median value and standard deviations were obtained based on histological examinations. Accordingly, epidural fibrosis decreased significantly in the study group compared to the control group. There was no statistically significant difference between the groups in terms of acute and chronic inflammation response and vascular proliferation.

Conclusion: The rESWT application immediately after surgery is effective in preventing epidural fibrosis after laminectomy in rats.

Keywords: Epidural fibrosis, laminectomy, radial extracorporeal shock wave therapy, rat.

the cellular mechanisms of epidural fibrosis and preventing epidural fibrosis after surgery has increased in recent years. ${ }^{[2,4-9]}$

Extracorporeal shock wave therapy (ESWT) is a non-invasive treatment method using sound waves to stimulate tissue healing. There is a growing number of studies in the literature, since shock wave therapy has been used for lithotripsy in the field of urology and, currently, it is frequently used in the treatment of many musculoskeletal diseases, chronic soft tissue 
wounds, neurological pathologies, andrological disorders, and ischemic heart diseases. ${ }^{[10]}$

Mechanical stress caused by high amplitude sound waves (shock waves) applied to tissue with ESWT cause morphological and physiological changes at the cellular level. The signals generated due to mechanical stimulation result in cytoskeleton rearrangement, expression of different genes, and changes in the expression levels of extracellular matrix (ECM) proteins, and cell proliferation and differentiation. ${ }^{[11]}$ Two types of ESWT are used in clinical medical treatment: focused (f) and radial (r) ESWT. Previous studies have shown that both ESWT applications improve healing by acting on fibroblasts. ${ }^{[11,2]}$

In recent years, the effect of ESWT on hypertrophic wounds and fibrosis has been examined in the literature. ${ }^{[13-15]}$ Data from both animal experiments and clinical applications have demonstrated that ESWT reduces hypertrophic wound formation and fibrosis. ${ }^{[14,15]}$ Considering these data, we hypothesized that rESWT treatment would reduce epidural fibrosis after laminectomy in rats. In this study, therefore, we aimed to investigate the effect of rESWT treatment in the prevention of epidural fibrosis after laminectomy in rats.

\section{MATERIALS AND METHODS}

All experimental procedures and care of the experimental animals were carried out in accordance with the European Communities Council Directive of November 24, 1986 (86/609/EEC) related to the protection of animals for experimental use.
The experiments were started after the approval of Başkent University Animal Experiments Ethics Committee (DA19/28). All experiments were carried out under the supervision of a veterinary physician at the Başkent University Laboratory of Animal Experiments. Eighteen 16-month-old male Sprague-Dawley rats weighing $300 \mathrm{~g}$ were used were used in this experimental study between November 2019 and February 2020. The rats were randomly divided into two groups as the control group ( $n=9$, L3-L4 total laminectomy without any treatment) and the study group ( $\mathrm{n}=9$, L3-L4 total laminectomy plus rESWT [MASTERPULS ${ }^{\circledR}$ MP100; Storz Medical AG, Kreuzlingen, Switzerland] used in orthopedics and traumatology clinic after laminectomy). The rESWT was applied three times a week for a total of six weeks with 200 pulse shock waves at a frequency of $3 \mathrm{~Hz}$, as described previously. ${ }^{[16]}$ Energy flux densities ranged from 0.01 to $0.23 \mathrm{~mJ} / \mathrm{mm}^{2}(14.3 \mathrm{MPa})$ at a constant pressure level of 3 bar. Each treatment session lasted approximately $75 \mathrm{sec}$ in proportion to the number of shots under standard conditions. The treatment was carried out by the clinician who also performed this application in patients in daily clinical practice. The subjects were kept in appropriately sized cages at a room temperature of $25^{\circ} \mathrm{C}$ in a 12-h light, 12-h dark cycle. No food or water restrictions were applied.

The subjects were fasted overnight before anesthesia. As an anesthetic method, intraperitoneal ketamine $60 \mathrm{mg} / \mathrm{kg}$ (Ketalar ${ }^{\mathrm{TM}}$, Pfizer, Sandwich, Kent, UK) and xylazine $7 \mathrm{mg} / \mathrm{kg}$ (Rompun ${ }^{\circledR} \%$ Bayer HealthCare AG, Leverkusen, Germany) were applied. Anesthesia was kept at a level that could not respond to pain, while spontaneous breathing of the rats
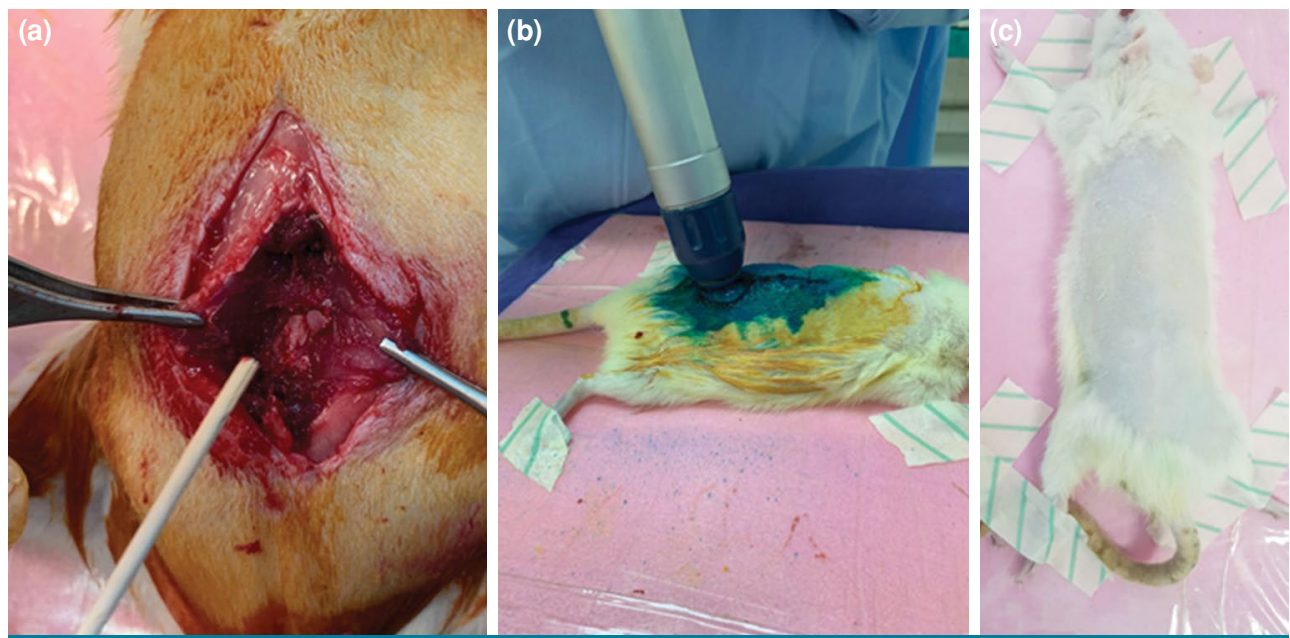

FIGURE 1. (a) Laminectomy. (b) Radial extracorporeal shock wave therapy application. (c) Appearance of surgical field at sixth week. 
continued. After the anesthesia application, the back area of the rats was shaved, and the prone position was placed in a way to fix the four limbs. Povidone-iodine (Betadine $^{\circledR}$, Avrio Health L.P, Stanford, USA) was used for local antisepsis. All surgical procedures were carried out by a single surgeon who was an expert in spinal surgery. A 3-cm skin incision was performed in the lower lumbar region. Following subperiosteal blunt dissection of the paravertebral muscles, L3-L4 laminae were reached. Laminectomy was performed with the help of a rongeur. Dura was exposed. Care was taken not to damage the dura during surgical manipulations. Abundant irrigation was performed with warm physiological saline. After hemostasis, the subcutaneous tissues were primed with $3 / 0$ Vicryl and the skin was closed with 3/0 Vicryl sutures (Figure 1). The rats were given intraperitoneal fentanyl $0.02 \mathrm{mg} / \mathrm{kg}$ (Fentanyl; Janssen-Cilag Pty Ltd, North Ryde, Australia) as an analgesic after the procedure. Intraperitoneal enrofloxacin $10 \mathrm{mg} / \mathrm{kg}$ (Baytril ${ }^{\oplus}$, Bayer HealthCare AG, Leverkusen, Germany) was administered for surgical infection prophylaxis. In the postoperative follow-up, the bladder of the rats was emptied regularly.

At the end of the sixth week, the rats in both groups were placed under general anesthesia in prone position, and all limbs were immobilized. The former skin incision was opened. The paravertebral muscle was opened by blunt dissection. The defective area undergoing lamination was detected. The vertebral column was removed from the proximal and distal approximately $0.5 \mathrm{~cm}$ of the laminectomy area, and the vertebral column was totally removed and placed in a $10 \%$ neutral buffered formalin solution. All rats were sacrificed using ketamine $100 \mathrm{mg} / \mathrm{kg}$ (Ketalar ${ }^{\mathrm{TM}}$, Sandwich, Kent, UK).

The specimens were fixed in 10\% buffered formalin for one week and, then, decalcified for four days in ethylenediaminetetraacetic acid/hydrochloric acid solution. After tissue samples were dehydrated with alcohol, they were processed and embedded in paraffin and $3-\mu \mathrm{m}$ sections were obtained. Tissue sections were stained with hematoxylin-eosin per protocol to determine the amount of acute and chronic inflammation and vascular proliferation. Histological evaluation was performed on a five-point grading system: (0) indicates the laminectomy defect was free of the parameter; (1) indicates a parameter of less than $25 \%$ of the laminectomy defect; (2) indicates a parameter of $50 \%$ of the laminectomy defect; (3) indicates a parameter of $75 \%$ of the laminectomy defect; and (4) indicates a parameter covering the entirety of the laminectomy side.

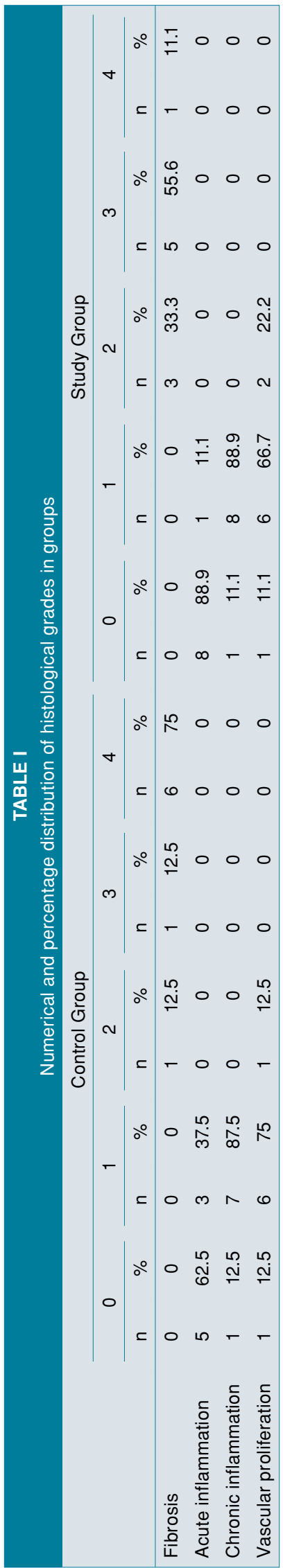




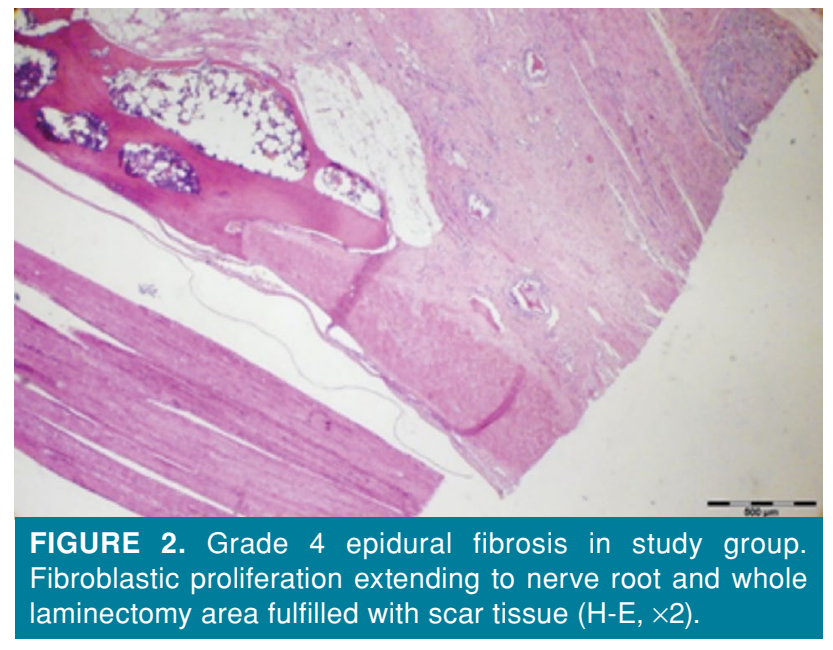

Hematoxylin-eosin staining was used in the evaluation of epidural fibrosis. Epidural fibrosis was graded according to following classification: (0) absence of fibrosis; (1) Fibrosis not adherent to the dural sac or nerve roots, less than $25 \%$ involvement; (2) Fibrosis adherent to the dural sac or nerve roots, less than 50\% involvement; (3) Fibrosis adherent to the dural sac and nerve roots, less than 75\% involvement; (4) Fibrosis throughout the vertebral canal, encompassing the dural sac and nerve roots, up to $100 \%$ involvement. ${ }^{[17]}$ The preparations were evaluated under Olympus BX50 light microscope (Olympus, Tokyo, Japan) by a single pathologist who was blinded to the method applied to the subjects used in the study.

\section{Statistical analysis}

Statistical analysis was performed using the SPSS version 16.0 software (SPSS Inc., Chicago, IL, USA). Descriptive data were expressed in mean \pm standard deviation, number and percentage. The Mann-Whitney $U$ test was used to compare the two groups. A $p$ value of $<0.05$ was considered statistically significant.
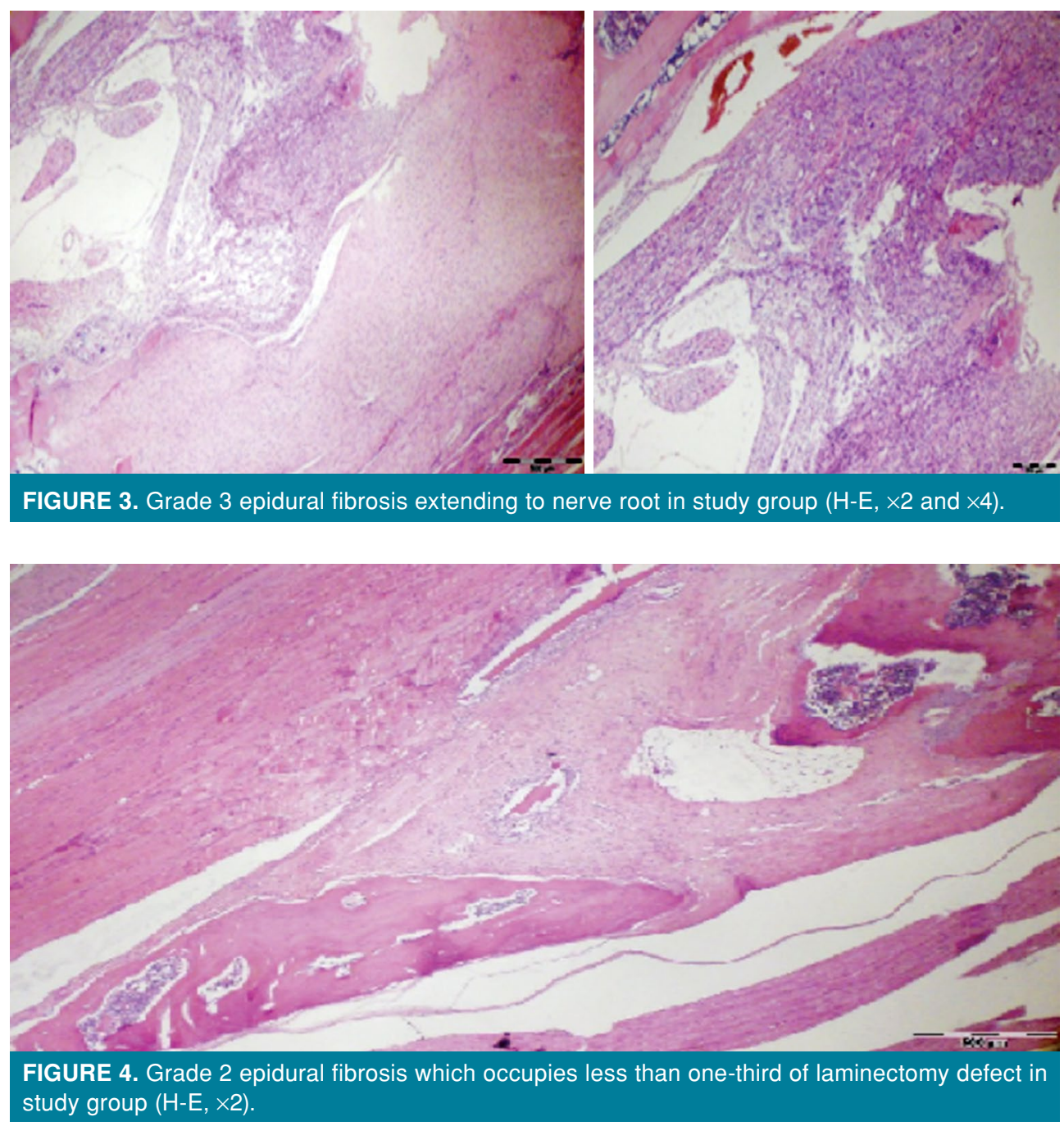


\begin{tabular}{|c|c|c|c|}
\hline \multicolumn{4}{|c|}{ TABLE II } \\
\hline & Control Group & Study Group & \\
\hline & Mean \pm SD & Mean $\pm S D$ & $p$ \\
\hline Fibrosis & $3.6 \pm 0.7$ & $2.8 \pm 0.7$ & $0.026^{*}$ \\
\hline Acute inflammation & $0.4 \pm 0.5$ & $0.1 \pm 0.3$ & 0.225 \\
\hline Chronic inflammation & $0.9 \pm 0.4$ & $0.9 \pm 0.3$ & 0.935 \\
\hline Vascular proliferation & $1 \pm 0.5$ & $1.1 \pm 0.6$ & 0.694 \\
\hline
\end{tabular}

\section{RESULTS}

After the surgical procedure, a rat in the control group was lost due to an anesthetic complication. No side effects were observed in the wound area and peripher al tissues related to the application of rESWT during the treatment process. Additionally, no laminectomyrelated complications such as infection, hematoma and cerebrospinal fluid leak were observed.

The histological examination results are shown in Table I. In the control group, Grade 4 epidural fibrosis was observed in six (75\%), Grade 3 in one $(12.5 \%)$, and Grade 2 in one (12.5\%) rat. In the study group, Grade 4 epidural fibrosis was detected in one (11.1\%) (Figure 2), Grade 3 in five (55.6\%) (Figure 3), and Grade 2 in three $(33.3 \%)$ rats (Figure 4). The mean value and standard deviations obtained as a result of evaluating the data obtained from histological examinations and the results of statistical analysis are given in Table II. Accordingly, epidural fibrosis decreased significantly in the study group compared to the control group $(p<0.05)$. On the other hand, there was no statistically significant difference between the groups in terms of acute and chronic inflammation response and vascular proliferation (Figures 5 and 6) ( $>00.05$ ).

\section{DISCUSSION}

Epidural fibrosis develops as a result of the natural wound healing process. The frequency of complications such as dura damage, nerve root damage, epidural hematoma, and infection increases in patients undergoing redo surgery due to scar tissue and adhesions caused by epidural fibrosis in the healing area. Therefore, studies to prevent epidural fibrosis formation in recent years have gained importance. ${ }^{[3,9]}$

Wound healing consists of four phases including hemostasis, inflammation, proliferation, and maturation, respectively. The vascular endothelial growth factor (VEGF) released in the early period of wound healing provides the migration of cells to the surgical field by increasing the vascular permeability and enhancing neovascularization. Due to the presence of mediators and cytokines in this area secreted from inflammatory cells, structural changes in the normal tissue may occur. ${ }^{[18]}$ Cytokines






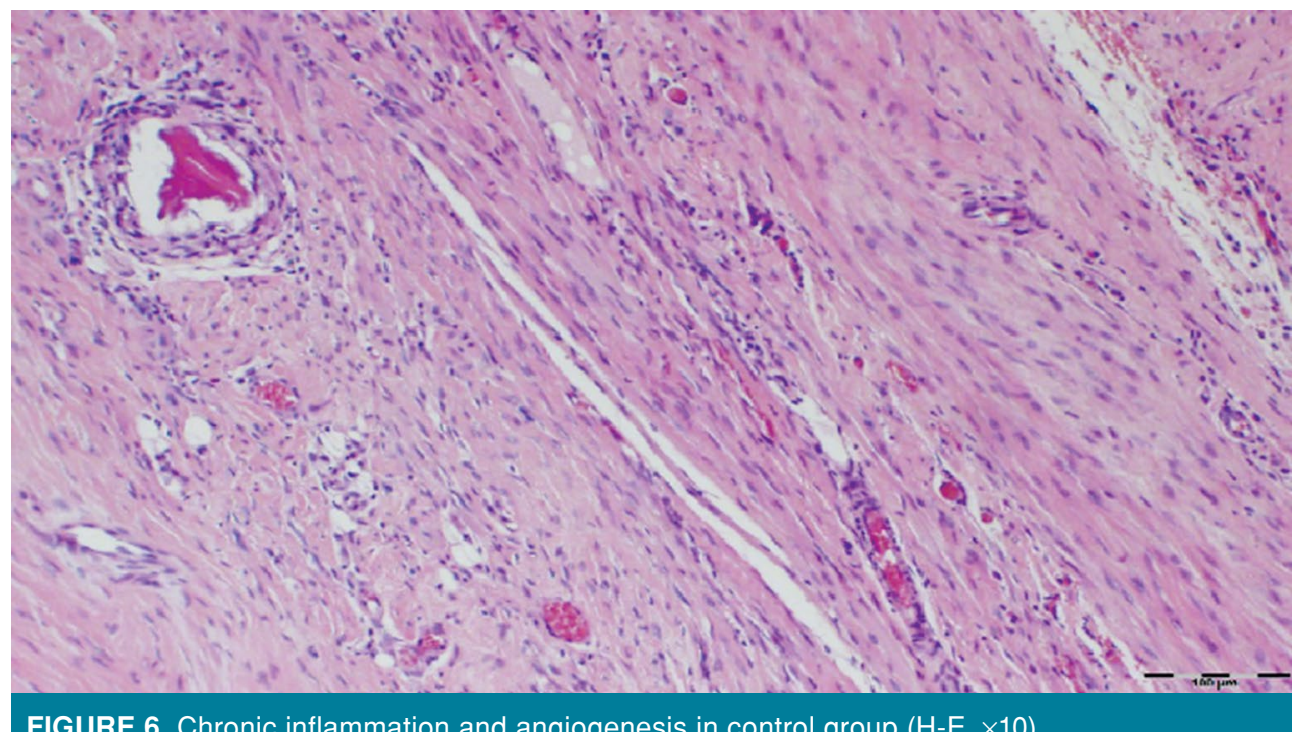

released into the environment in epidural fibrosis such as interleukin (IL)-6, IL-8, transforming growth factor beta (TGF- $\beta$ ), and tumor necrosis factor alpha (TNF- $\alpha$ ) induce the pathological process by affecting the fibroblasts and, thereby, leading to the formation of granulation tissue in the hematoma in the surgical area. $^{[3]}$

Previous studies have shown that fibroblasts are the main cell type which play a role in the pathogenesis of epidural fibrosis. The proliferation of fibroblasts that migrate after paraspinal muscle dissection and bone resection in the epidural space, the differentiation of myofibroblasts, and the accumulation of fibronectin and ECM secreted from myofibroblasts cause the formation of fibrosis. In addition, TGF-1 $\beta$ released from fibroblasts increases the formation of fibrosis by causing a positive feedback on the fibrotic process. ${ }^{[4,9]}$

There are many studies in the literature using a wide range of agents and affecting these molecular steps. Each treatment method used in these studies shows its main effect by affecting different steps of fibrosis formation. Based on the studies examining the effects of hemostasis and vascular proliferation on the development of epidural fibrosis, studies on treatment methods to reduce bleeding and neovascularization are at the forefront. Tural Emon et al. $^{[2]}$ applied hemostatic polysaccharide agent to rats after laminectomy and showed that this agent had no effect on the development of epidural fibrosis. On the other hand, in studies evaluating the effects of ranibizumab ${ }^{[9]}$ and sorafenib, ${ }^{[19]}$ VEGF inhibitors, on rats, both agents significantly reduced epidural fibrosis.

Among the studies regarding the inflammatory response phase, the effect of specific inhibition of inflammatory cytokines and the use of anti-inflammatory agents on epidural fibrosis have been frequently examined in the literature. For the inhibition of specific inflammatory cytokines, etanercept, a TNF- $\alpha$ blocker, ${ }^{[1]}$ tamoxifen, a TGF- $\beta$ blocker, $^{[7]}$ methyl palmitate, ${ }^{[18]}$ and dexmedetomidine ${ }^{[4]}$ have been extensively investigated. Also, there are studies using diclofenac sodium ${ }^{[20]}$ and curcumin ${ }^{[21]}$ as anti-inflammatory agents. All these studies showed that both inflammatory cytokine inhibition and the use of anti-inflammatory agents significantly reduced the development of epidural fibrosis by producing anti-inflammatory and antioxidant effects.

The inflammation phase of wound healing has a direct effect on fibroblast proliferation and wound maturation. The ECM components are synthesized and stored by the myofibroblasts during the remodeling process of wound healing, while they are degraded by matrix metalloproteinases (MMPs) and collagenases to provide ECM rearrangement. In severe inflammatory conditions where the balance between synthesis and degradation is disrupted, TGF- $\beta$ expression increases and, thereby, increasing the fibroblast proliferation. The large amount of ECM stored by the growing fibroblasts causes fibrosis. ${ }^{[15,22]}$ Therefore, researchers have also examined the effects of fibroblast proliferation and wound maturation on epidural fibrosis development. In previous studies, 
hyaluronic acid-based gels, ${ }^{[6]}$ platelet-rich fibrin, and ADCON-L ${ }^{\circledR}$ gel $^{[3]}$ were used to create an anti-fibrotic mechanical barrier. Although the methods that create a mechanical barrier show different efficacy, they significantly reduce the development of epidural fibrosis. In addition, several studies are available in the literature reporting significantly reduced epidural fibrosis, using agents such as pentoxifylline ${ }^{[23]}$ with an anti-fibroblastic effect, and diltiazem, ${ }^{[20]}$ an fibroblast growth factor-2 inhibitor.

In the current study, we evaluated the effect of rESWT on epidural fibrosis after laminectomy in rats. Our study results showed that rESWT significantly reduced epidural fibrosis without affecting acute and chronic inflammatory response and vascular proliferation. ${ }^{[2]}$ Therefore, we believe that rESWT exerts its main protective effect against epidural fibrosis through fibroblast proliferation and wound maturation. In vitro studies in which hypertrophic scar formation was observed demonstrated the effect of rESWT administration on fibroblasts. ${ }^{[15,22]}$ In a study on fibroblasts from human hypertrophic scars, Cui et al. ${ }^{[2]}$ reported that rESWT administration reduced TGF- $\beta$ expression with an anti-fibrotic effect by significantly reducing ECM components such as type-1 collagen and fibronectin. In another study, Zhao et al. ${ }^{[15]}$ created a hypertrophic wound model in rabbits and examined the effect of ESWT application. The authors showed that both low- and high-energy rESWT application reduced fibroblast density and suppressed hypertrophic wound formation. These findings are consistent with our study results. Considering these data, we believe that rESWT exerts its positive effect with its impact on ECM organization and signaling.

Radial ESWT application is a cost-effective and easy method which can be applied safely in the clinical practice. Studies on chronic wounds have shown that this treatment method increases wound healing. ${ }^{[12]}$ Moreover, in these studies, rESWT administration is applied directly to the areas where the skin integrity is lost by direct contact. ${ }^{[25]}$ Therefore, we believe that initiating rESWT in the early postoperative period in clinical practice would both reduce epidural fibrosis and positively affect wound healing without any adverse effects. Considering the possible side effects of systemic drugs that have been shown to have a positive effect on the prevention of epidural fibrosis, we expect that the use of rESWT in orthopedic practice would increase day by day as a result of studies showing the positive effects of rESWT. In addition, this non-invasive method can be used in combination with other systemic and local treatment methods after revision surgeries where the risk of epidural fibrosis is further increased. On the other hand, for humans, the application head of the shock wave generator can be changed, allowing sound waves to access deeper tissues. Thus, an effective application of rESWT would be provided in patients with different subcutaneous tissue thicknesses.

The main limitation of this study is its small sample size. In addition, the effects of rESWT application on different pressures and pulse numbers were unable to be examined. The effect of rESWT application, on wound healing, was not evaluated. On the other hand, the main strength of the present study is that the expression levels of cellular biomarkers showing inflammation and vascular proliferation were evaluated. Furthermore, in this study, the histopathological changes at the postoperative sixth week were evaluated, and the effect of a longer follow-up period on histopathological findings was not examined. Finally, the Masson's trichrome staining, another staining method showing histopathological fibrosis, was unable to be used for histopathological evaluation due to financial reasons.

In conclusion, rESWT application immediately after surgery is effective in preventing epidural fibrosis after laminectomy in a rat model. To the best of our knowledge, this is the first experimental study to demonstrate the effect of rESWT application on epidural fibrosis in rats, which has different areas of use in clinic practice, such as muscletendon pathologies, chronic soft tissue wounds, kidney diseases, cardiac, and neurological problems. Nonetheless, further large-scale studies are needed to demonstrate the effects of rESWT application on epidural fibrosis at the cellular level and to evaluate its clinical application and results in humans.

\section{Declaration of conflicting interests}

The authors declared no conflicts of interest with respect to the authorship and/or publication of this article.

\section{Funding}

The authors received no financial support for the research and/or authorship of this article.

\section{REFERENCES}

1. Kizilay Z, Cetin NK, Topcu A, Ismailoglu O, Omurlu IK, Aktas S, et al. Effect of etanercept on the formation of epidural fibrosis in an experimental model. Turk Neurosurg 2018;28:111-7.

2. Tural Emon S, Somay H, Orakdogen M, Uslu S, Somay A. Effects of hemostatic polysaccharide agent on epidural fibrosis formation after lumbar laminectomy in rats. Spine J 2016;16:414-9. 
3. Demirel E, Yildiz K, Çadirci K, Aygün H, Şenocak E, Gündoğdu B. Effect of platelet-rich fibrin on epidural fibrosis and comparison to ADCON ${ }^{\circledR}$ Gel and hyaluronic acid. Acta Orthop Traumatol Turc 2018;52:469-74.

4. Yaman ME, Ergül G, Güvenç Y, Öztürk Y, Erbay FK, Tolunay T, et al. Evaluation of topical Dexmedetomidine administration in postlaminectomy epidural fibrosis rat model. Int J Surg 2018;53:80-5.

5. Bryant MS, Bremer AM, Nguyen TQ. Autogeneic fat transplants in the epidural space in routine lumbar spine surgery. Neurosurgery 1983;13:367-70.

6. Hu MH, Yang KC, Sun YH, Chen YC, Yang SH, Lin FH. In situ forming oxidised hyaluronic acid/adipic acid dihydrazide hydrogel for prevention of epidural fibrosis after laminectomy. Eur Cell Mater 2017;34:307-20.

7. Ozturk Y, Bozkurt I, Yaman ME, Guvenc Y, Tolunay T, Bayram $\mathrm{P}$, et al. Histopathologic analysis of tamoxifen on epidural fibrosis. World Neurosurg 2018;111:e941-e8.

8. Prusick VR, Lint DS, Bruder WJ. Cauda equina syndrome as a complication of free epidural fat-grafting. A report of two cases and a review of the literature. J Bone Joint Surg [Am] 1988;70:1256-8.

9. Yilmaz A, Karatay M, Yildirim T, Celik H, Sertbas I, Erdem Y, et al. Prevention of epidural fibrosis using ranibizumab in a postlaminectomy rat model. Turk Neurosurg 2017;27:119-23.

10. Zhang J, Kang N, Yu X, Ma Y, Pang X. Radial extracorporeal shock wave therapy enhances the proliferation and differentiation of neural stem cells by Notch, PI3K/AKT, and Wnt/ $\beta$-catenin Signaling. Sci Rep 2017;7:15321.

11. Frairia R, Berta L. Biological effects of extracorporeal shock waves on fibroblasts. A review. Muscles Ligaments Tendons J 2012;1:138-47.

12. Schmitz C, Császár NB, Milz S, Schieker M, Maffulli N, Rompe JD, et al. Efficacy and safety of extracorporeal shock wave therapy for orthopedic conditions: a systematic review on studies listed in the PEDro database. Br Med Bull 2015;116:115-38.

13. Fischer S, Mueller W, Schulte M, Kiefer J, Hirche C, Heimer S, et al. Multiple extracorporeal shock wave therapy degrades capsular fibrosis after insertion of silicone implants. Ultrasound Med Biol 2015;41:781-9.

14. Saggini R, Saggini A, Spagnoli AM, Dodaj I, Cigna E, Maruccia $M$, et al. Extracorporeal shock wave therapy: An emerging treatment modality for retracting scars of the hands. Ultrasound Med Biol 2016;42:185-95.
15. Zhao JC, Zhang BR, Hong L, Shi K, Wu WW, Yu JA. Extracorporeal shock wave therapy with low-energy flux density inhibits hypertrophic scar formation in an animal model. Int J Mol Med 2018;41:1931-8.

16. Yahata K, Kanno H, Ozawa H, Yamaya S, Tateda S, Ito K, et al. Low-energy extracorporeal shock wave therapy for promotion of vascular endothelial growth factor expression and angiogenesis and improvement of locomotor and sensory functions after spinal cord injury. J Neurosurg Spine 2016;25:745-55.

17. Temel SG, Ozturk C, Temiz A, Ersozlu S, Aydinli U. A new material for prevention of epidural fibrosis after laminectomy: oxidized regenerated cellulose (interceed), an absorbable barrier. J Spinal Disord Tech 2006;19:270-5.

18. Kizilay Z Assistant Professor, Kahraman Cetin N. Effect of methyl palmitate on the formation of epidural fibrosis in an experimental epidural fibrosis Model. J Invest Surg 2018;31:469-74.

19. Tanriverdi O, Erdogan U, Tanik C, Yilmaz I, Gunaldi $\mathrm{O}$, Adilay $\mathrm{HU}$, et al. Impact of sorafenib on epidural fibrosis: An immunohistochemical study. World J Clin Cases 2018;6:249-58.

20. Erdogan B, Is M, Aker FV, Emon ST, Engin T, Akar EA, et al. Preventative effect of diclofenac sodium and/or diltiazem in rats with epidural fibrosis. Bratisl Lek Listy 2019;120:813-8.

21. Ismailoglu O, Kizilay Z, Cetin NK, Topcu A, Berber O. Effect of curcumin on the formation of epidural fibrosis in an experimental laminectomy model in rats. Turk Neurosurg 2019;29:440-4.

22. Cui HS, Hong AR, Kim JB, Yu JH, Cho YS, Joo SY, et al. Extracorporeal shock wave therapy alters the expression of fibrosis-related molecules in fibroblast derived from human hypertrophic scar. Int J Mol Sci 2018;19:124.

23. Kelten B, Erdogan H, Antar V, Sanel S, Tuncdemir M, Kutnu $\mathrm{M}$, et al. Pentoxifylline inhibits epidural fibrosis in postlaminectomy rats. Med Sci Monit 2016;22:840-7.

24. Atik OŞ. What are the expectations of an editor from a scientific article? Jt Dis Relat Surg 2020;31:597-8.

25. Aschermann I, Noor S, Venturelli S, Sinnberg T, Mnich CD, Busch C. Extracorporal shock waves activate migration, proliferation and inflammatory pathways in fibroblasts and keratinocytes, and improve wound healing in an open-label, single-arm study in patients with therapy-refractory chronic leg ulcers. Cell Physiol Biochem 2017;41:890-906. 